Huchon, P., Taylor, B., and Klaus, A. (Eds.)

Proceedings of the Ocean Drilling Program, Scientific Results Volume 180

\section{QUATERNARY SiLICEOUS SPONGE SPICULES IN THE WESTERN WOODLARK BAsIN, SOUTHWESt PACIFIC (ODP LEG 180) ${ }^{1}$}

\author{
Eugenio Andri, ${ }^{2}$ Stefania Gerbaudo, ${ }^{2}$ and Massimiliano Testa ${ }^{2}$
}

${ }^{1}$ Andri, E., Gerbaudo, S., and Testa, M., 2001. Quaternary siliceous sponge spicules in the western Woodlark Basin, southwest Pacific (ODP Leg 180). In Huchon, P., Taylor, B., and Klaus, A. (Eds.), Proc. ODP, Sci. Results, 180, 1-8 [Online]. Available from World Wide Web: <http://www-odp. tamu.edu/publications/180_SR/ VOLUME/CHAPTERS/153.PDF>. [Cited YYYY-MM-DD]

${ }^{2}$ Dipartimento per lo Studio del Territorio e delle sue Risorse, Università degli Studi di Genova, Corso Europa, 26-16132 Genova, Italy. Correspondence author: gerbaudo@box.tin.it; gerbaudo@dipteris.unige.it; and gerbaudo@noos.fr

Initial receipt: 25 October 2000 Acceptance: 23 March 2001

Web publication: 10 August 2001

Ms 180SR-153 


\section{MATERIALS AND METHODS}

The samples and preparations employed in this study are the same as those employed in our radiolarian analysis (Testa et al., this volume). A total of 78 sediment samples were studied from cores recovered from the Quaternary sediments.

Tables T1 and T2 show the distribution of the siliceous sponge spicules in Holes 1108B, 1109C, 1110A, 1111A, 1112A, 1114A, 1115A, and $1115 \mathrm{~B}$. The relative abundance is indicated by the following scale:

$$
\begin{aligned}
A & =\text { abundant }(>100 \text { spicules }) \\
\mathrm{C} & =\text { common }(10-100 \text { spicules }) \\
\mathrm{U} & =\text { uncommon }(1-9 \text { spicules }) \\
\mathrm{N} & =\text { not found }(0 \text { spicules })
\end{aligned}
$$

\section{SPONGE SPICULES}

The terminology employed and the determinations done on the sponge spicules found in the samples refer to de Laubenfels (1955), McCartney (1990), Ahlbach and McCartney (1992), Zolnik et al. (1992), and Lurvey et al. (1998).

\section{Monaxons (spicules with a single axis)}

Diactinal (monaxons with similar ends)

Oxea (pointed at both ends) (Pl. P1, fig. 9): very abundant in almost all the samples studied. Usually represents $40 \%-90 \%$ of the spicules observed. As observed by McCartney (1987), this form shows great variability in the length of the axis $(80-350 \mu \mathrm{m})$.

Acanthoxea (Oxea more or less covered by spines) (Pl. P1, fig. 8): only two specimens were found at Site 1108.

Strongyle (monaxon with rounded ends) (Pl. P1, fig. 3): few in almost all samples investigated except for Site 1112 where it is absent entirely.

Acanthostrongyle (Strongyle more or less covered by spines): uncommon, found in only three samples from Sites 1108, 1109, and 1110. Specimens similar to the ones found in this study are illustrated by Lurvey et al., 1998 (pl. 1, fig. 3).

\section{Monactinal (monaxon rounded at one end and pointed at the other end)}

Style (monactinal monaxon with one end blunt and the other pointed) (Pl. P1, fig. 5): together with Oxea, this is the most abundant form found during this study; absent at Site 1114.

Acanthostyle (Style more or less covered by spines): uncommon yet found at all sites except for Hole 1115A; total of 13 specimens.

Tylostyle (pointed at one end with a knob at the rounded end): found only in some samples but with quite good frequency (10\%-20\% of the assemblage). The specimens observed are generally similar to the ones illustrated by McCartney, 1990 (pl. 2, fig. 6).
T1. Distribution of siliceous sponge spicules, Holes 1108B, 1109C, 1110A, 1111A, 1112A, and $1114 \mathrm{~A}$, p. 6.

T2. Distribution of siliceous sponge spicules, Holes 1115A and 1115B, p. 7.

P1. Siliceous sponge spicules, p. 8.

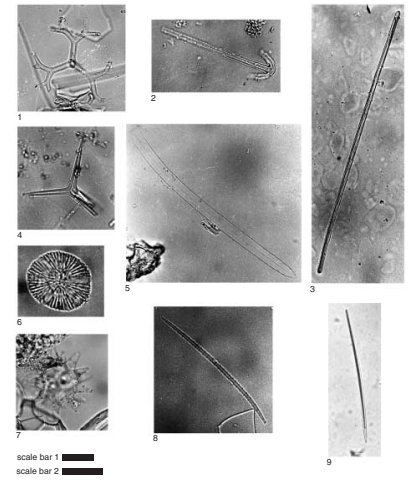




\section{E. ANDri Et AL.

Triaxons (spicules with three axes)

Triod (spicule with three similar rays): uncommon; found at all sites except for Sites 1110 and 1114. See Lurvey et al., 1998 (pl. 2, fig. 7) as an example. Spiny forms were found also: Triod (spined).

Pentacts (Triaxon with five rays, four of which are usually arranged in one plane): only three specimens were found at Sites 1108 and 1111. These forms were also described during studies of Leg 120, Site 748 (Ahlbach and McCartney, 1992).

Pinnule (Pentacts with long spines on the dissimilar ray): only one specimen was found at Site 1108. See also Ahlbach and McCartney (1992).

\section{Tetraxons (spicules with four axes)}

Calthrops (spicule with equal rays arranged normal to faces of the tetrahedron) (Pl. P1, fig. 4): uncommon, found at Sites 1108, 1109, 1111, and 1114. Spiny forms were found too: Calthrops (spined).

Triaene (Tetraxon with one ray different from three other similar rays): according to the morphology presented by the three similar rays, four varieties were recognized:

Anatriaene (Triaene with the three similar rays curving back toward the point of the odd ray) (Pl. P1, fig. 2): four specimens were recovered at Sites 1108, 1109, and 1110. See McCartney, 1990 (pl. 1, fig. 2).

Dichotriaene (Triaene with forked rays) (Pl. P1, fig. 1): uncommon and broken specimens were recovered only at Sites 1109, 1111, and 1115 (Hole 1115B). This species was also found by McCartney, 1990 (pl. 2, figs. 5, 7).

Plagiotriaene (Triaene with the three similar rays thick and perpendicular to the odd ray): only two specimens were observed in samples recovered at Sites 1108 and 1111. These forms were also described during studies of Leg 101 samples (Palmer, 1988).

Protriaene (Triaene with the three similar rays curving away from the point of the odd ray): only one specimen was observed at Site 1111. See also Palmer, 1988 .

\section{Polyaxons}

Spheraster (spicule with rays diverging from a globular center) (Pl. P1, fig. 7): nine specimens were found in five samples at Sites 1109, 1111, and 1115 (Hole 1115B).

Sterraster (ovoid spicule with numerous blunt rays) (Pl. P1, fig. 6): usually uncommon. Fifteen specimens were found in seven samples from Sites 1109, 1111, and 1115 (Hole 1115B). The same species was found by Palmer (1988) and Bukry (1979).

\section{Sigmas}

Sigma-C (spicule shaped like the letter " $C$ "): four specimens were found-one specimen at Site 1108, one at Site 1111, and two specimens at Site 1112. Similar specimens to the ones found in this study are illustrated by McCartney, 1987 (pl. 5, figs. 1, 5).

\section{ACKNOWLEDGMENTS}

S. Gerbaudo thanks the Italian Secretariat for financial support to participate in Leg 180 and carry out this research. This work was also supported by funds to E. Andri (University of Genova). Thanks also to 
E. ANDRI ET AL.

QuATERnARY Siliceous SPONGE SPICULES

K. McCartney and the ODP editorial staff for their reviews of this paper and R. Laronga, who corrected our English. 


\section{REFERENCES}

Ahlbach, W.J., and McCartney, K., 1992. Siliceous sponge spicules from Site 748. In Wise, S.W., Jr., Schlich, R., et al., Proc. ODP, Sci. Results, 120: College Station, TX (Ocean Drilling Program), 833-837.

Bukry, D., 1979. Coccolith and silicoflagellate stratigraphy, northern Mid-Atlantic Ridge and Reykjanes Ridge, Deep Sea Drilling Project Leg 49. In Luyendyk, B.P., Cann, J.R., et al., Init. Repts. DSDP, 49: Washington (U.S. Govt. Printing Office), 551-581.

Laubenfels, M.W. de, 1955. Porifera. In Moore, R.C. (Ed.), Treatise on Invertebrate Paleontology: Lawrence (Univ. Kansas Press), part E:21-112.

Lurvey, L.K., McCartney, K., and Wei, W., 1998. Siliceous sponge spicules, silicoflagellates, and ebridians from Hole 918D, continental rise of the Greenland Margin. In Saunders, A.D., Larsen, H.C., and Wise, S.W., Jr. (Eds.), Proc. ODP, Sci. Results, 152: College Station, TX (Ocean Drilling Program), 191-199.

McCartney, K., 1987. Siliceous sponge spicules from Deep Sea Drilling Project Leg 93. In van Hinte, J.E., Wise, S.W., Jr., et al., Init. Repts. DSDP, 93 (Pt. 2): Washington (U.S. Govt. Printing Office), 815-824.

— 1990. Siliceous sponge spicules from Ocean Drilling Program Leg 113. In Barker, P.F., Kennett, J.P., et al., Proc. ODP, Sci. Results, 113: College Station, TX (Ocean Drilling Program), 963-970.

Palmer, A., 1988. Paleoenvironmental significance of siliceous sponge spicules from Sites 627 and 628, Little Bahama Bank, Ocean Drilling Program Leg 101. In Austin, J.A., Jr., Schlager, W., et al., Proc. ODP, Sci. Results, 101: College Station, TX (Ocean Drilling Program), 159-168.

Zolnik, R., McCartney, K., and White, L.D., 1992. Siliceous sponge spicules from Site 795. In Pisciotto, K.A., Ingle, J.C., Jr., von Breymann, M.T., Barron, J., et al., Proc. ODP, Sci. Results, 127/128 (Pt. 1): College Station, TX (Ocean Drilling Program), 541-544. 


\section{E. ANDri ET AL. \\ QuATERnARY Siliceous Sponge SPICUles}

Table T1. Distribution of siliceous sponge spicules, Holes 1108B, 1109C, 1110A, 1111A, $1112 \mathrm{~A}$, and 1114A.

\begin{tabular}{|c|c|c|c|c|c|c|c|c|c|c|c|c|c|c|c|c|c|c|c|c|c|c|}
\hline $\begin{array}{l}\text { Core, section, } \\
\text { interval }(\mathrm{cm})\end{array}$ & $\begin{array}{l}\text { Depth } \\
\text { (mbsf) }\end{array}$ & 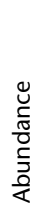 & 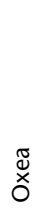 & 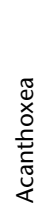 & 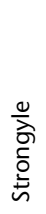 & 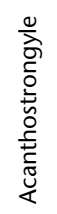 & $\stackrel{0}{\stackrel{2}{\lambda}}$ & 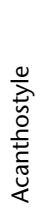 & 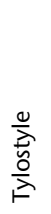 & 을 & 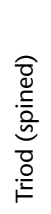 & 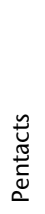 & $\begin{array}{l}\frac{d}{\vec{D}} \\
\stackrel{\underline{D}}{\underline{L}}\end{array}$ & $\begin{array}{l}\frac{\breve{O}}{0} \\
\frac{\underline{\underline{E}}}{\mathrm{E}} \\
\text { U. }\end{array}$ & 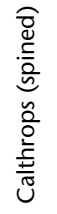 & 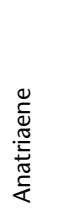 & 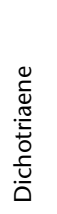 & 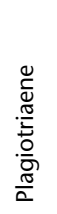 & 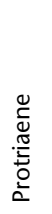 & 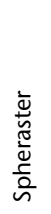 & 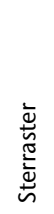 & 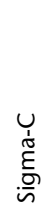 \\
\hline \multicolumn{23}{|l|}{ 180-1108B- } \\
\hline $1 \mathrm{R}-1,10.0-12.0$ & 0.10 & A & $x$ & & $x$ & $\mathrm{x}$ & $x$ & $\mathrm{x}$ & $x$ & $x$ & & & $\mathrm{x}$ & & & & & & & & & $\mathrm{x}$ \\
\hline $1 \mathrm{R}-2,50.0-52.0$ & 1.50 & A & $x$ & $x$ & $x$ & & $x$ & $\mathrm{x}$ & $x$ & $x$ & $x$ & $\mathrm{x}$ & & $\mathrm{x}$ & $x$ & $x$ & & $\mathrm{x}$ & & & & \\
\hline $8 R-1,18.0-20.0$ & 62.88 & u & $x$ & & & & $x$ & & & & & & & & & & & & & & & \\
\hline $8 \mathrm{R}-1,55.0-57.0$ & 63.25 & $\mathrm{u}$ & $x$ & & & & & & & & & & & & & & & & & & & \\
\hline $9 \mathrm{R}-1,20.0-22.0$ & 72.50 & $\mathrm{u}$ & $\mathrm{x}$ & & & & $\mathrm{x}$ & & & & & & & & & & & & & & & \\
\hline 9R-1, 101.0-103.0 & 73.31 & u & $x$ & & & & $x$ & & & & & & & & & & & & & & & \\
\hline 9R-1, 115.0-117.0 & 73.45 & $\mathrm{~N}$ & & & & & & & & & & & & & & & & & & & & \\
\hline $10 \mathrm{R}-1,80.0-82.0$ & 82.70 & $\mathrm{~N}$ & & & & & & & & & & & & & & & & & & & & \\
\hline $14 \mathrm{R}-1,73.0-75.0$ & 120.93 & $\mathrm{~N}$ & & & & & & & & & & & & & & & & & & & & \\
\hline $14 \mathrm{R}-1,67.0-69.0$ & 122.03 & $\mathrm{~N}$ & & & & & & & & & & & & & & & & & & & & \\
\hline $15 \mathrm{R}-2,35.0-37.0$ & 130.98 & $\mathrm{~N}$ & & & & & & & & & & & & & & & & & & & & \\
\hline $15 \mathrm{R}-2,85.5-87.0$ & 131.49 & $\mathrm{~N}$ & & & & & & & & & & & & & & & & & & & & \\
\hline $16 \mathrm{R}-2,30.0-32.0$ & 141.20 & N & & & & & & & & & & & & & & & & & & & & \\
\hline $16 \mathrm{R}-2,104.0-105.0$ & 141.94 & U & & & $\mathrm{x}$ & & & & & & & & & & & & & & & & & \\
\hline \multicolumn{23}{|l|}{ 180-1109C- } \\
\hline $2 \mathrm{H}-2,43.0-45.0$ & 9.33 & $\mathrm{~N}$ & & & & & & & & & & & & & & & & & & & & \\
\hline $2 \mathrm{H}-4,37.0-39.0$ & 12.03 & $\mathrm{~N}$ & & & & & & & & & & & & & & & & & & & & \\
\hline $3 \mathrm{H}-1,40.0-42.0$ & 17.30 & $\mathrm{~N}$ & & & & & & & & & & & & & & & & & & & & \\
\hline $3 \mathrm{H}-1,49.5-50.5$ & 17.40 & $\mathrm{U}$ & & & $x$ & & & & & & & & & & & & & & & & & \\
\hline $3 \mathrm{H}-6,104.0-106.0$ & 25.44 & $\mathrm{~N}$ & & & & & & & & & & & & & & & & & & & & \\
\hline $4 \mathrm{H}-1,58.0-60.0$ & 26.98 & $\mathrm{u}$ & $x$ & & & & & & & & & & & $\mathrm{x}$ & $\mathrm{x}$ & & & & & & & \\
\hline $4 \mathrm{H}-4,83.5-86.0$ & 31.74 & $\mathrm{u}$ & & & $x$ & & & & & & & & & & & & & & & & & \\
\hline $5 \mathrm{H}-3,85.0-86.0$ & 39.75 & U & $x$ & & $x$ & & & & & & & & & $\mathrm{x}$ & & & & & & & & \\
\hline $5 \mathrm{H}-5,29.0-31.0$ & 42.19 & C & $x$ & & $x$ & & $x$ & & $x$ & $\mathrm{x}$ & & & & $\mathrm{x}$ & & & $x$ & & & & $x$ & \\
\hline $5 \mathrm{H}-6,38.0-39.0$ & 43.78 & $\mathrm{u}$ & & & & & & & & & & & & & & & & & & $x$ & & \\
\hline $6 \mathrm{H}-5,24.0-26.0$ & 51.64 & $\mathrm{~N}$ & & & & & & & & & & & & & & & & & & & & \\
\hline $7 \mathrm{H}-3,23.0-25.0$ & 58.13 & C & $\mathrm{x}$ & & $x$ & & $x$ & $x$ & & & & & & & & $x$ & $x$ & & & & & \\
\hline 7H- $4,118.0-120.0$ & 60.58 & $\mathrm{u}$ & & & & & & & & & & & & & & & & & & $\mathrm{x}$ & & \\
\hline $8 \mathrm{H}-6,126.0-126.0$ & 73.16 & $\mathrm{~N}$ & & & & & & & & & & & & & & & & & & & & \\
\hline $9 \mathrm{H}-7,50.0-52.5$ & 83.04 & $\mathrm{u}$ & & & $x$ & & $x$ & & & & & & & & & & & & & & & \\
\hline $10 \mathrm{H}-5,13.0-15.0$ & 89.53 & $\mathrm{~N}$ & & & & & & & & & & & & & & & & & & & & \\
\hline $11 \mathrm{H}-6,81.0-83.0$ & 101.21 & u & & & $x$ & & $x$ & & & & & & & & & & & & & & & \\
\hline $12 X-4,80.0-82.0$ & 107.7 & U & $\mathrm{x}$ & & $\mathrm{x}$ & $x$ & $\mathrm{x}$ & & & & & & & & & & & & & & & \\
\hline $12 X-6,74.0-76.0$ & 110.64 & u & & & & & $x$ & & $x$ & & & & & & & & & & & & & \\
\hline $13 X-5,38.0-40.0$ & 118.38 & $\mathrm{u}$ & $x$ & & & & & & & & & & & & & & & & & & & \\
\hline $14 \mathrm{X}-1,7.5-10.0$ & 121.68 & $\mathrm{~N}$ & & & & & & & & & & & & & & & & & & & & \\
\hline $16 X-4,59.0-60.0$ & 145.89 & u & $x$ & & $x$ & & & & & & & & & & & & & & & & & \\
\hline $20 X-4,43.0-45.0$ & 184.23 & $\mathrm{~N}$ & & & & & & & & & & & & & & & & & & & & \\
\hline $23 X-7,60.0-62.0$ & 216.35 & U & $x$ & & & & & & & & & & & & & & & & & & & \\
\hline \multicolumn{23}{|l|}{ 180-1110A- } \\
\hline $1 \mathrm{H}-2,77.0-79.0$ & 2.27 & C & $x$ & & & & $x$ & & $x$ & & & & & & & & & & & & & \\
\hline $1 \mathrm{H}-4,94.0-96.0$ & 5.44 & C & $x$ & & & & $\mathrm{x}$ & & $\mathrm{x}$ & & & & & & & $\mathrm{x}$ & & & & & & \\
\hline $2 \mathrm{H}-1,58.0-62.0$ & 7.58 & C & $x$ & & $x$ & $x$ & $\mathrm{x}$ & & & & & & & & & & & & & & & \\
\hline $2 \mathrm{H}-1,81.0-85.0$ & 7.81 & C & $x$ & & $x$ & & $x$ & $\mathrm{x}$ & & & & & & & & $\mathrm{x}$ & & & & & & \\
\hline \multicolumn{23}{|l|}{ 180-1111A- } \\
\hline $8 \mathrm{R}-1,77.0-81.0$ & 68.47 & $\mathrm{~N}$ & & & & & & & & & & & & & & & & & & & & \\
\hline $8 R-3,29.0-31.0$ & 70.99 & $\mathrm{~N}$ & & & & & & & & & & & & & & & & & & & & \\
\hline $11 \mathrm{R}-1,44.0-46.0$ & 97.14 & A & $x$ & & $x$ & & $x$ & $x$ & $x$ & $x$ & & & & $x$ & & & $x$ & & $x$ & $x$ & & \\
\hline $13 \mathrm{R}-2,21.0-23.0$ & 117.37 & u & & & & & $x$ & & & & & & & & & & & & & & $\mathrm{x}$ & \\
\hline $14 \mathrm{R}-1,60.0-62.0$ & 126.40 & C & $\mathrm{x}$ & & $x$ & & $x$ & & & & & & & & & & & & & & & \\
\hline $14 \mathrm{R}-3,62.0-64.0$ & 129.42 & A & $\mathrm{x}$ & & $\mathrm{x}$ & & $\mathrm{x}$ & $x$ & $\mathrm{x}$ & & & & & & & & $x$ & & & $\mathrm{x}$ & $x$ & \\
\hline $15 \mathrm{R}-1,38.0-40.0$ & 135.78 & C & $x$ & & $x$ & & $x$ & & $x$ & & & $x$ & & & & & & & & & & \\
\hline $16 \mathrm{R}-1,5.0-7.0$ & 145.05 & C & $\mathrm{x}$ & & $\mathrm{x}$ & & $\mathrm{x}$ & $\mathrm{x}$ & $\mathrm{x}$ & & & & & & & & $\mathrm{x}$ & $\mathrm{x}$ & & & & $\mathrm{x}$ \\
\hline \multicolumn{23}{|l|}{$180-1112 \mathrm{~A}-$} \\
\hline 1R-1, 100.0-101.0 & 1.00 & C & $\mathrm{x}$ & & & & $x$ & $\mathrm{x}$ & & $\mathrm{x}$ & & & & & & & & & & & & $x$ \\
\hline \multicolumn{23}{|l|}{ 180-1114A- } \\
\hline 1R-CC, 23.5-25.0 & 0.23 & C & $x$ & & $x$ & & & $x$ & $x$ & & & & & $x$ & & & & & & & & \\
\hline
\end{tabular}

Notes: $\mathrm{A}=$ abundant, $\mathrm{C}=$ common, $\mathrm{U}=$ uncommon, $\mathrm{N}=$ not present. $\mathrm{X}=$ present. 
E. ANDri Et AL.

QuATERnARY Siliceous SPonge SPICULES

Table T2. Distribution of siliceous sponge spicules, Holes 1115A and 1115B.

\begin{tabular}{|c|c|c|c|c|c|c|c|c|c|c|c|c|c|c|c|c|c|c|c|c|c|c|}
\hline $\begin{array}{l}\text { Core, section, } \\
\text { interval }(\mathrm{cm})\end{array}$ & $\begin{array}{l}\text { Depth } \\
\text { (mbsf) }\end{array}$ & $\begin{array}{l}\stackrel{8}{ } \\
\frac{1}{\pi} \\
\frac{0}{0} \\
\frac{5}{2} \\
\frac{0}{<}\end{array}$ & 忍 & 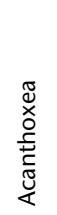 & 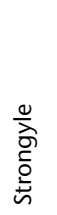 & 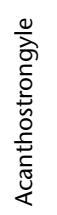 & $\frac{0}{i}$ & 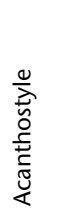 & $\begin{array}{l}\frac{0}{\lambda} \\
\frac{\tilde{\Delta}}{\lambda} \\
\end{array}$ & $\underset{\frac{0}{2}}{\circ}$ & 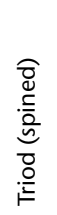 & 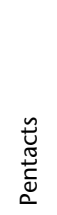 & $\begin{array}{l}\frac{d}{\vec{T}} \\
\stackrel{\underline{c}}{\underline{c}}\end{array}$ & 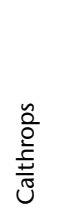 & 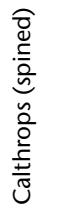 & 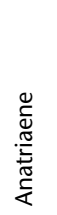 & 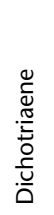 & 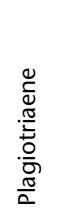 & 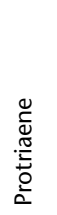 & 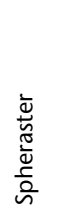 & 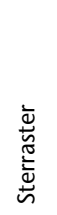 & 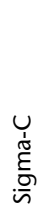 \\
\hline \multicolumn{23}{|l|}{ 180-1115A- } \\
\hline $1 \mathrm{H}-2,5.0-7.0$ & 0.33 & $\mathrm{U}$ & $x$ & & $\mathrm{x}$ & & & & $x$ & & & & & & & & & & & & & \\
\hline $1 \mathrm{H}-4,4.5-6.5$ & 3.05 & $\mathrm{~N}$ & & & & & & & & & & & & & & & & & & & & \\
\hline \multicolumn{23}{|l|}{ 180-1115B- } \\
\hline $1 \mathrm{H}-1,5.0-7.0$ & 0.05 & C & $\mathrm{x}$ & & $\mathrm{x}$ & & $\mathrm{x}$ & & & & & & & & & & & & & & $\mathrm{x}$ & \\
\hline $1 \mathrm{H}-3,27.0-29.0$ & 3.27 & C & $\mathrm{x}$ & & & & & & & & & & & & & & & & & & $\mathrm{x}$ & \\
\hline $2 \mathrm{H}-4,145.0-147.0$ & 13.15 & C & $\mathrm{x}$ & & $x$ & & & & & & & & & & & & $\mathrm{x}$ & & & & & \\
\hline $2 \mathrm{H}-6,130.0-132.0$ & 16.00 & $\mathrm{u}$ & $\mathrm{x}$ & & & & $x$ & & & & & & & & & & & & & & & \\
\hline $3 \mathrm{H}-2,58.0-60.0$ & 18.78 & $\mathrm{C}$ & $x$ & & $x$ & & $x$ & $x$ & $x$ & & & & & & & & & & & & & \\
\hline $4 \mathrm{H}-4,59.0-62.0$ & 31.29 & $\mathrm{C}$ & $\mathrm{x}$ & & $x$ & & $x$ & $x$ & $x$ & & & & & & & & & & & & & \\
\hline $4 \mathrm{H}-6,66.0-68.0$ & 34.36 & C & $\mathrm{x}$ & & $x$ & & $x$ & & $x$ & $\mathrm{x}$ & & & & & & & $\mathrm{x}$ & & & $\mathrm{x}$ & $x$ & \\
\hline $5 \mathrm{H}-1,19.0-21.0$ & 35.89 & C & $\mathrm{x}$ & & $\mathrm{x}$ & & $\mathrm{x}$ & & $\mathrm{x}$ & & & & & & & & & & & & & \\
\hline $5 \mathrm{H}-4,70.0-72.0$ & 40.90 & C & $x$ & & & & $\mathrm{x}$ & & $\mathrm{x}$ & & & & & & & & & & & & & \\
\hline $6 \mathrm{H}-2,53.0-55.0$ & 47.23 & C & $\mathrm{x}$ & & & & $x$ & & $x$ & & & & & & & & & & & & & \\
\hline $6 \mathrm{H}-5,55.0-57.0$ & 51.75 & $\mathrm{C}$ & $x$ & & $\mathrm{x}$ & & $x$ & & $x$ & & & & & & & & & & & & & \\
\hline $6 \mathrm{H}-7,39.0-43.0$ & 54.59 & $\mathrm{C}$ & $\mathrm{x}$ & & $x$ & & $x$ & & & & & & & & & & & & & & $x$ & \\
\hline $7 \mathrm{H}-4,77.0-79.0$ & 59.97 & C & $\mathrm{x}$ & & $\mathrm{x}$ & & $x$ & & & & & & & & & & & & & & & \\
\hline $7 \mathrm{H}-6,78.0-80.0$ & 62.98 & C & $x$ & & $x$ & & & $\mathrm{x}$ & & & & & & & & & & & & & & \\
\hline $8 \mathrm{H}-3,111.0-113.0$ & 68.31 & C & $\mathrm{x}$ & & $\mathrm{x}$ & & $\mathrm{x}$ & & $x$ & & & & & & & & & & & & & \\
\hline $8 \mathrm{H}-6,17.0-19.0$ & 71.87 & C & $\mathrm{x}$ & & $\mathrm{x}$ & & & & & $\mathrm{x}$ & & & & & & & $\mathrm{x}$ & & & & & \\
\hline $9 \mathrm{H}-1,5.0-7.0$ & 73.75 & C & $\mathrm{x}$ & & $x$ & & $x$ & & & & & & & & & & & & & & & \\
\hline $9 \mathrm{H}-3,64.0-66.0$ & 77.34 & C & $\mathrm{x}$ & & $x$ & & & & & $\mathrm{x}$ & & & & & & & & & & & & \\
\hline $10 \mathrm{H}-7,14.0-16.5$ & 92.34 & C & $\mathrm{x}$ & & & & $x$ & & & & & & & & & & & & & & & \\
\hline $11 \mathrm{H}-3,21.0-23.0$ & 95.91 & $\mathrm{C}$ & $x$ & & & & $x$ & & $x$ & & & & & & & & & & & & & \\
\hline $11 \mathrm{H}-5,8.0-15.0$ & 98.78 & C & & & $\mathrm{x}$ & & $x$ & & & & & & & & & & & & & & & \\
\hline $11 \mathrm{H}-7,9.0-11.0$ & 101.79 & C & $\mathrm{x}$ & & $\mathrm{x}$ & & $\mathrm{x}$ & & $\mathrm{x}$ & $\mathrm{x}$ & & & & & & & & & & & & \\
\hline $12 \mathrm{H}-1,17.0-19.0$ & 102.37 & C & $\mathrm{x}$ & & $x$ & & $\mathrm{x}$ & & & & & & & & & & & & & & & \\
\hline $12 \mathrm{H}-4,33.0-35.0$ & 107.03 & C & $\mathrm{x}$ & & & & $x$ & & $x$ & & & & & & & & & & & & & \\
\hline
\end{tabular}

Notes: $\mathrm{A}=$ abundant, $\mathrm{C}=$ common, $\mathrm{U}=$ uncommon, $\mathrm{N}=$ not present. $\mathrm{X}=$ present. 


\section{E. ANDri ET AL. \\ QUATERNARY Siliceous SPonge SPICUleS}

Plate P1. Siliceous sponge spicules from ODP Leg 180. 1. Dichotriaene (315×) (Sample 180-111A-11R-1, 44.0-46.0 cm). 2. Anatriaene (315×) (Sample 180-1110A-1A-4, 94.0-96.0 cm). 3. Strongyle (315×) (Sample 180-1108B-1R-1, 10.0-12.0 cm). 4. Calthrops (500×) (Sample 180-1111A-11R-1, 44.0-46.0 cm). 5. Style (315×) (Sample 180-1110A-1H-2, 77.0-79.0 cm). 6. Sterraster (500×) (Sample 180-1111A-14R-3, 62.0-64.0 cm). 7. Spheraster (500×) (Sample 180-1109C-7H-4, 118.0-120.0 cm). 8. Acanthoxea (315×) (Sample 1801108B-1R-2, 50.0-52.0 cm). 9. Oxea (315×) (Sample 180-1108B-1R-1, 10.0-12.0 cm). Scale bar 1 (for photos taken at $500 \times)=25 \mu \mathrm{m}$; scale bar 2 (for photos taken at $315 \times$ ) $=50 \mu \mathrm{m}$.
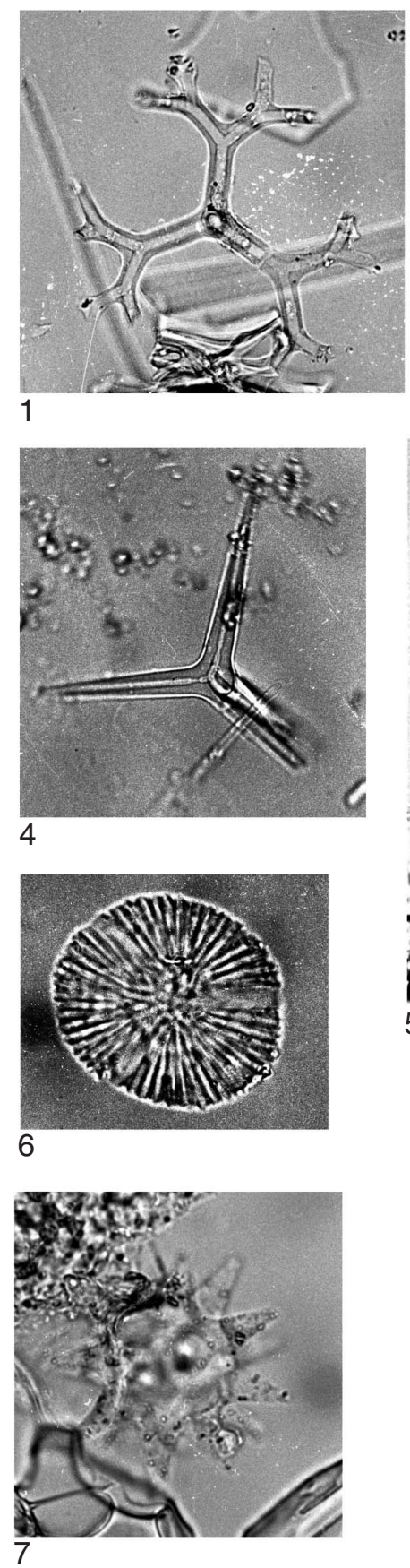

scale bar 1

scale bar 2
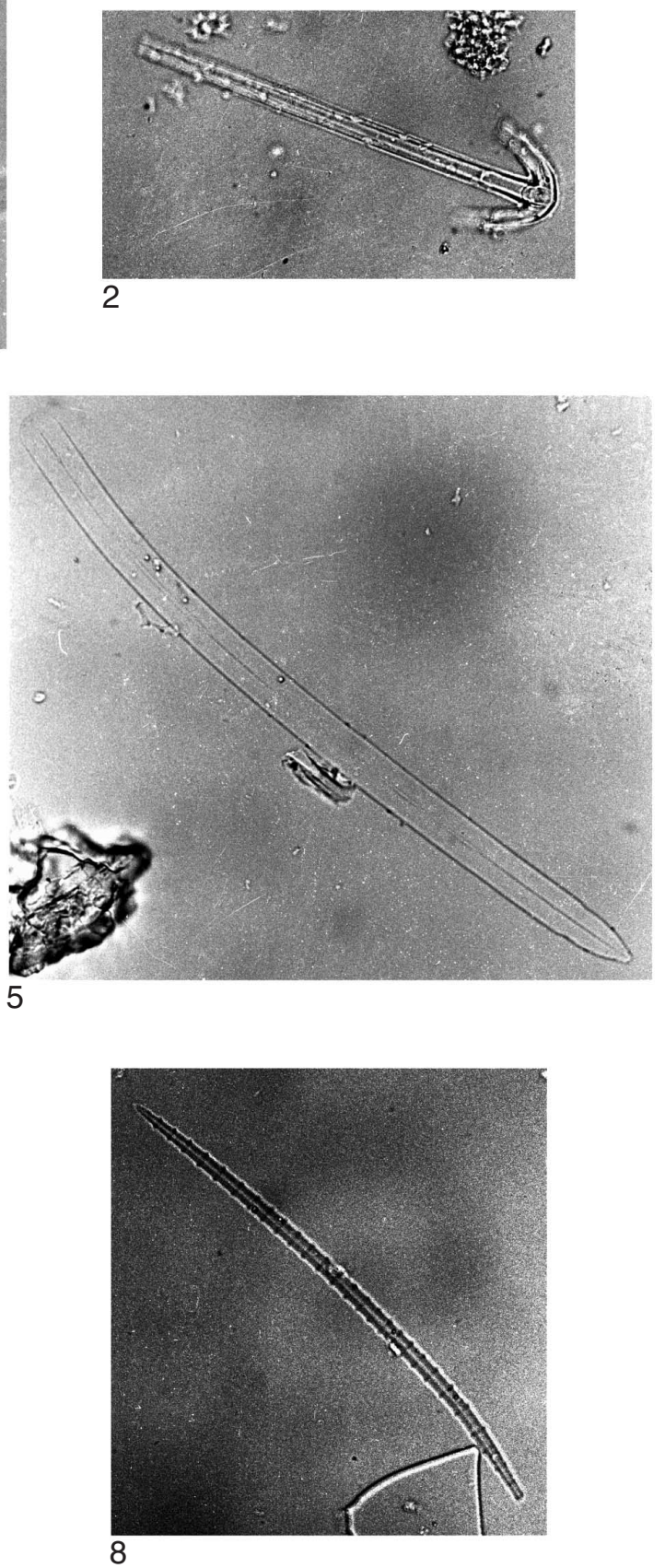

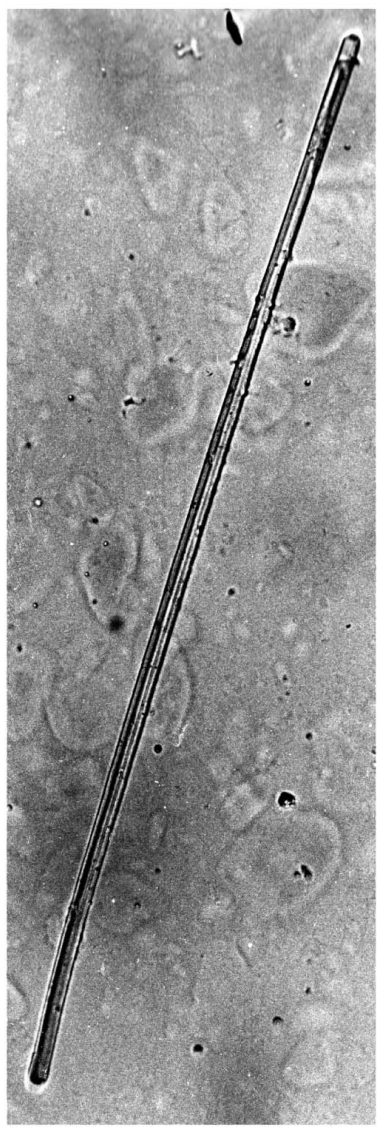

3

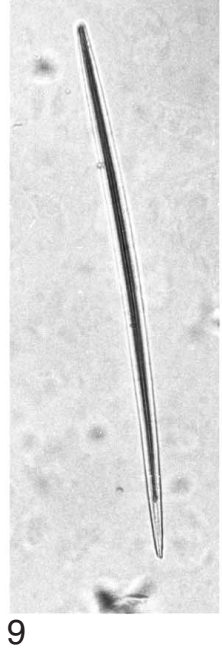

